\title{
Normalización y requisitos funcionales de la descripción archivística: una propuesta metodológica
}

\author{
Raquel Gómez Díaz \\ Raquel Bringas González \\ Universidad de Salamanca (España)
}

\section{Resumen}

Las nuevas tecnologías de la información y la comunicación exigen la normalización de los diferentes elementos informativos que hacen posible el acceso e intercambio de información. Para cumplir con estos objetivos es necesario conocer qué datos son realmente útiles para el intercambio, y normalizar solo estos. Se analizan los objetivos de la descripción archivística, tratando de responder a las preguntas de qué es, para qué, por qué, cómo y para quién son las descripciones archivísticas, se indican los distintos tipos de normas y se propone el modelo entidad-relación para el estudio de los requisitos funcionales de las descripciones archivísticas hechas con ISAD $(\mathrm{G})$ con el objetivo de ver qué elementos son útiles para el desarrollo de nuevas normas de descripción de contenido.

Palabras clave: Normas de descripción archivística. Descripción archivística. Normalización. Requisitos funcionales.

\section{Abstract}

The new technologies of information and communication (TIC) require the standardization of the different informative elements, to make possible the access and interchange of information. In order to fulfil this objective, the data that is really useful must be previously known. These elements and not others must be standardized. From this departing point, the general goals of the archival description are analysed, responding to the following questions: what is it, what is it for, why, how and whom the archival descriptions are directed to. The different available standards are reviewed and the entity-relationship model is adopted for modelling the functional requirements of $\operatorname{ISAD}(\mathrm{G})$-based archival descriptions. Several elements are detected that can be useful in the further normalization of content descriptions. 
Keywords: Archival standards. Archival descriptions. Standardizations. Functional requirements.

\section{Introducción}

En los archivos tenemos que hacer frente a un número de documentos que crece de manera exponencial, sin que el tiempo y los recursos que podemos dedicar a su tratamiento se incrementen en igual proporción. Sin embargo, aunque la experiencia de compartir información en el entorno archivístico se ve condicionada por el carácter singular de los documentos, hoy en día ya casi nadie duda entre los profesionales de que el trabajo realizado por un archivero sí es aprovechable por otros. De aquí surge la necesidad de trabajar con formatos normalizados para que la información contenida en los archivos se pueda, además de compartir, intercambiar.

La normalización de los elementos informativos contenidos en la descripción archivística implica conocer qué datos son realmente útiles a la hora de intercambiar, para que sean estos y no otros los que normalicemos. Necesitamos hacer una revisión de qué es lo que queremos comunicar, para qué y por qué hacerlo, cómo lo vamos a llevar a cabo y para quién vamos a normalizar las descripciones del contenido de los archivos. De esta manera, podremos realizar con éxito el intercambio y la comunicación de información.

Con el fin de mostrar cómo se pueden estudiar los requisitos funcionales de la descripción archivística y que estos aporten la información necesaria para el desarrollo de las normas de contenido, aplicaremos el modelo entidad-relación. Daremos unas breves notas sobre los comienzos de los trabajos de normalización y el panorama actual de las principales normas nacionales e internacionales de descripción. Se propondrá la utilización de una tabla de análisis para obtener una valoración que nos ayude a continuar el desarrollo normativo.

\section{La descripción como acceso a la información}

El conjunto de documentos reunidos, creados y/o acumulados por una institución o persona en el ejercicio de sus actividades, se custodian, organizan y difunden en razón de su importancia como garantía de derechos y su interés informativo para la toma de decisiones, la historia o la investigación científica.

Para posibilitar el acceso a toda esta documentación es imprescindible la existencia de un enlace entre la información contenida en los documentos y el usuario. El archivero tendrá que analizar los fondos y los documentos archivísticos para sintetizar y condensar la información en ellos contenida, generando descripciones - representaciones de las unidades documentales - para ofrecerlas a los interesados (Heredia, 1991). Por tanto, no podemos perder de vista que uno

Scire. $11: 1$ (en.-jun. 2005) 103-112. ISSN 1135-3761. 
de los fines de la descripción es proporcionar al usuario una vía útil de acceso a la información. Si tenemos en cuenta que el perfil del usuario de archivo ha ido evolucionando con la sociedad, la comunidad archivística deberá tratar de conciliar necesidades informativas tan dispares como las planteadas por investigadores, ciudadanos o usuarios de la administración virtual.

\section{Los trabajos de normalización archivística}

La llegada de la tecnología informática al mundo de los archivos abrió nuevas posibilidades y al mismo tiempo supuso la necesidad de un replanteamiento en la manera de trabajar. El intercambio obliga a utilizar patrones comunes, desencadenando un proceso de normalización en la década de los setenta.

Los archiveros estadounidenses se lanzaron a esta aventura y tras varios intentos se dieron cuenta de que necesitaban establecer categorías iguales donde introducir elementos con datos equiparables. Tomando como base las AngloAmerican Cataloging Rules (AACR2), elaboraron las primeras normas de descripción, los Archives Personal Papers and Manuscripts (APPM). Poco después y como producto del consenso de la comunidad archivística canadiense, surgen las Rules for Archival Description (RAD), cuyo proceso de redacción de carácter integrador ha garantizado su implantación.

En la normalización británica Michael Cook es el responsable de la primera redacción del Manual of Archival Description (MAD), manual donde, como él mismo expresa, no pretende innovar, sino tan solo sintetizar y codificar lo más posible la práctica tradicional (Bonal, 2002). Posteriormente y recogiendo las distintas opiniones profesionales vertidas al respecto, reelabora el manual junto con Margaret Procter, dando lugar al MAD2. La principal aportación de este manual fue la fijación de los niveles descriptivos, propuesta que quedará recogida en la ISAD(G).

La iniciativa de estos tres países propició el comienzo de los trabajos de normalización a nivel internacional, promoviéndose desde el Consejo Internacional de Archivos (CIA) la creación de normas y reglas que faciliten el acceso, la gestión y el intercambio de información en los archivos (CIA, 1993). Desde los orígenes han estado presentes las distintas realidades nacionales. Por ello, teniendo en cuenta la dificultad de aunar políticas archivísticas diferentes, se ha tratado de que exista la máxima participación de los distintos países.

\section{Tipos de normas}

Los análisis y estudios realizados por Berman (1993), Walch (1994), Fox (1994) y Santamaría (2003) clasifican las normas de descripción en cuatro categorías. Siguiendo este esquema, tenemos primeramente las normas de estructura

Scire. $11: 1$ (en.-jun. 2005) 103-112. ISSN 1135-3761. 
(1), en las que se establecen los elementos de las distintas representaciones. También necesitamos unas normas de contenido (2) que nos indiquen cómo vamos a presentar los elementos de información, teniendo en cuenta que en algunos casos se podrá precisar exactamente la información que hay que incluir mediante las normas de valores (3). Por último, para hacer que otros puedan consultar y aprovechar nuestro trabajo, tendremos que tratar de estudiar cómo vamos a intercambiar y comunicar esta información, desarrollando para ello las normas de intercambio o comunicación de datos (4).

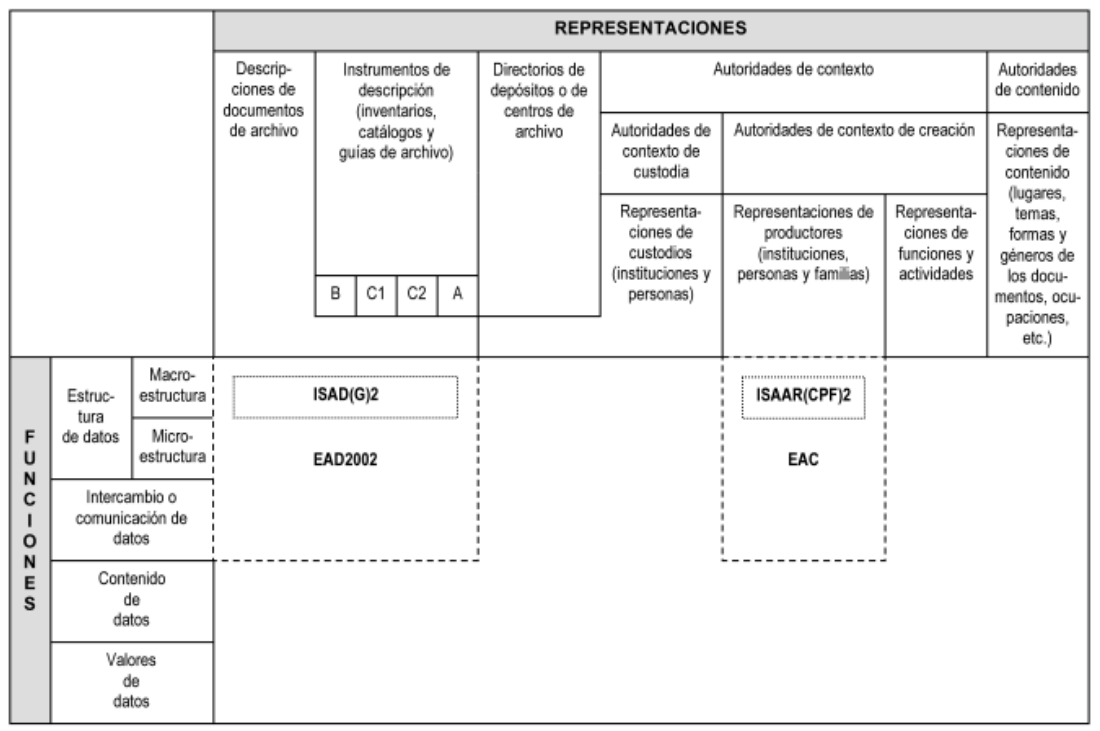

Figura 1. Esfuerzos normalizadores en el campo de la archivística (Santamaría, 2003)

Reflexionando sobre las necesidades y las herramientas desarrolladas en los distintos intentos de normalizar la descripción, Abelardo Santamaría (2003) nos presenta gráficamente qué es lo que hay hecho y qué es lo que queda por hacer en el panorama internacional (figura 1).

Tenemos definida una norma de macroestructura de datos, el International Standard Archival Description (General) - ISAD $(G)$-, internacionalmente aceptada, y otra no tan implantada, que sirve para la representación de los productores y autoridades de contexto de creación, el International Standard Archival Authority Record for Corporate Bodies, Persons and Families - ISAAR(CPF) - . También contamos con la Encoded Archival Description (EAD), que nació como norma para facilitar el intercambio de información, pero definió una estructura 
más específica que la que estaba definida previamente en la ISAD(G). Por eso, al mismo tiempo que una norma de intercambio, es una norma de estructura, con un alto grado de especificidad.

Como podemos apreciar, el proceso normalizador no está concluido y parece que el siguiente paso lógico será trabajar en los desarrollos nacionales de normas de contenido de datos, partiendo siempre de ISAD(G) y EAD (5). En nuestro caso, la archivística española no es ajena a este proceso, contamos con el Manual de descripción multinivel (2001), auspiciado por la Junta de Castilla y León, y con las tareas que está realizando el Grupo de Trabajo coordinado desde la Subdirección General de los Archivos Estatales.

\section{El modelo entidad-relación en la descripción}

La propia ISAD $(G)$, en su glosario de términos, define la descripción como "una fiel representación de una unidad de descripción y las partes que la componen, si existen, mediante la captura, análisis, organización y registro de la información, que sirve para identificar, gestionar, localizar y explicar (6) los materiales archivísticos, y el contexto y sistemas documentales que los han producido. También describe el proceso" (ISAD(G), 1999).

Según la definición que acabamos de ver, el término hace referencia tanto al proceso como al resultado. En cuanto al proceso, el CIA nos indica cómo hacemos la descripción - capturando, analizando, organizando y registrando la información que contiene el archivo- y desarrolla en la norma cuál debe ser el resultado del proceso.

Vista la ambivalencia existente en la definición, necesitamos saber cómo podemos nombrar y qué es exactamente ese producto. Una posible denominación es la representación de la unidad de descripción (en adelante RUD), que podemos definir como el conjunto de elementos informativos referidos a una unidad archivística, resultado del proceso de descripción, y que sirve tanto para representar, identificar y recuperar la información como para proporcionar el acceso al soporte de dicha información.

Como ya hemos visto, para hacer la descripción contamos con una norma de estructura. La ISAD $(\mathrm{G})$ tiene 26 elementos, 6 obligatorios y el resto optativos, cuya combinación tiene que proporcionarnos la identificación, gestión, localización y contextualización de los fondos, series y documentos. De este modo conseguiremos que la RUD sea pertinente (que cumpla con las funciones de la descripción), compatible (que no genere contradicciones con otras RUD) y explícita (que dé la información necesaria, sin datos repetidos o inapropiados).

Para ver esto de una manera más clara proponemos como posible vía de trabajo la aplicación del modelo entidad-relación con el fin de estudiar los requisitos

Scire. 11 : 1 (en.-jun. 2005) 103-112. ISSN 1135-3761. 
funcionales de la descripción, es decir, analizar si los elementos de la descripción archivística se ajustan a las funciones que se especifican en la norma. Este modelo está ampliamente extendido y contrastado, y sirve para la representación de objetos del mundo real. Ha sido aplicado con éxito en el diseño de bases de datos y en el estudio de los requisitos funcionales de los registros bibliográficos (Ríos, 2003), por citar algún ejemplo en materias afines.

\subsection{Definiciones previas: entidad, relación y requisito funcional}

Ahora bien, con caracter previo a la aplicación de este modelo, necesitamos aclarar los conceptos de entidad y relación dentro del marco específico de trabajo de la descripción archivística.

Por entidad entendemos cualquier clase de objeto distinguible en un sistema que comparte similares características o propiedades con otros. Estas entidades se caracterizan por los atributos que las describen y son comunes a los miembros de un conjunto de entidades; y además pueden ser usadas como una prueba de pertenencia (Amat, 1989). Las entidades no están aisladas sino que se relacionan con otras. En nuestro caso, la entidad es la RUD, que se corresponde con un nivel de descripción (fondo, sección, subsección, serie, subserie, unidad documental). Cada entidad tiene asociados una serie de atributos, a saber, los 26 elementos de la $\operatorname{ISAD}(\mathrm{G})$ que tienen que cumplir las funciones de la descripción y no tienen la misma importancia en todos los niveles. Si somos capaces de consensuar cuáles son los atributos principales asociados a cada una de las entidades, se habrá dado un paso importante en la normalización de la descripción.

Por su parte, las entidades se relacionan entre sí, ayudando a contextualizar la información. Podemos establecer dos tipos de relaciones: las verticales o de pertenencia - que relacionan el todo con las partes que la componen, es decir, la relación padre-hijo o, lo que es lo mismo, la relación que hay entre un fondo y las partes que componen ese fondo - y las horizontales - las que se producen entre las entidades que pertenecen a un mismo nivel, como es el caso de las distintas series que forman un fondo (Bonal, 2001) - . A su vez, estas entidades se relacionan con otras procedentes de otros tipos de representación de información, como pueden ser las representaciones de productores. Tener en cuenta todas estas relaciones es importante porque nos puede facilitar el trabajo de la descripción.

Finalmente, por requisito funcional entendemos la condición necesaria de un atributo para que cumpla una función determinada. En nuestro caso las funciones son aquellas que se recogen en la definición de la norma (identificar, gestionar, localizar y contextualizar), mientras que los atributos son los elementos que forman cada RUD, es decir, cada entidad de la descripción. 


\section{Propuesta metodológica}

Para hacer el estudio hemos diseñado una tabla de doble entrada donde se especifican los elementos que componen cada RUD. Proponemos analizar los atributos correspondientes a cada tipo de RUD, planteando en un principio el estudio de los requisitos funcionales desde un punto de vista estrictamente teórico. Podremos, a partir de la norma, construir un modelo con las funciones específicas que debería cumplir cada atributo, modelo que nos dará una base para la redacción de normas de contenido y para el desarrollo de las correspondientes normas de valores en los casos pertinentes. Aunque existe la posibilidad de establecer una escala que refleje los distintos grados de cumplimiento, optaremos por un sistema de valoración binario, eliminando una complejidad en el estudio que en realidad no aportaría datos especialmente enriquecedores.

Partiendo de las premisas revisadas, en un intento de aportar mayor claridad a la propuesta, presentamos la que podría ser una tabla tipo (tabla I, en la página siguiente) para el estudio de los requisitos funcionales de la descripción, en un entorno real. La tabla se divide en cuatro columnas, que se corresponden con las funciones de la descripción asignadas por la norma; mientras que en las filas se recogen los 26 elementos de la $\operatorname{ISAD}(\mathrm{G})$, estructurados por áreas para aportar una mayor claridad.

Esta tabla se aplicará a cada nivel, tomando a priori los niveles fondo, serie y unidad documental porque son los únicos en los que hay consenso entre las distintas normas nacionales (Carnicer, 1999), lo cual no impide que esta misma metodología se utilice para el estudio de otros niveles, si se considera pertinente. Para aplicar la tabla, tomaremos cada atributo de manera individual, valorando el cumplimiento de cada una de las funciones. La presencia de función la marcaremos con un 1; la ausencia, con un 0 . De este modo, por ejemplo, si consideramos, que el elemento "Código de referencia" debería servir para "identificar", asignaremos un 1 en la casilla correspondiente.

\section{Conclusiones}

El proceso normalizador no está concluido. Para continuar el desarrollo de la normativa, es necesario un estudio nacional pormenorizado de la $\operatorname{ISAD}(\mathrm{G})$, donde se analicen los requisitos funcionales de la descripción. Para llevarlo a cabo, nos parece interesante la aplicación del modelo entidad-relación, que nos puede proporcionar una visión estructurada de los elementos que conforman la representación de cada unidad documental y las relaciones existentes entre ellas, siempre dentro del contexto de la descripción multinivel recogido en la normativa internacional. En esta propuesta, este proceso se materializa en la creación de una tabla en la que aparecen reflejadas las funciones que debería cumplir cada elemento o atributo. 


\begin{tabular}{|c|c|c|c|c|c|}
\hline \multirow[t]{2}{*}{ Áreas } & \multirow[t]{2}{*}{ Elementos } & \multicolumn{4}{|c|}{ Funciones } \\
\hline & & 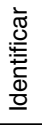 & 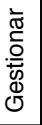 & 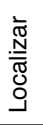 & 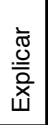 \\
\hline \multirow{5}{*}{ 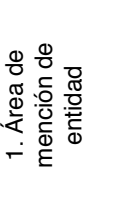 } & 1.1. Código de referencia & & & & \\
\hline & 1.2 Título & & & & \\
\hline & 1.3. Fechas y lugares & & & & \\
\hline & 1.4. Nivel de descripción & & & & \\
\hline & 1.5. Extensión y soporte & & & & \\
\hline \multirow{4}{*}{ 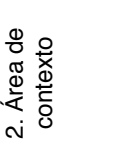 } & 2.1. Organismo productor & & & & \\
\hline & 2.2. Historia institucional & & & & \\
\hline & 2.3. Historia archivística & & & & \\
\hline & 2.4. Fuente inmediata de adquisición o transferencia & & & & \\
\hline \multirow{4}{*}{ 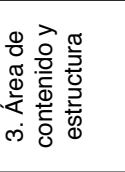 } & 3.1. Alcance y contenido & & & & \\
\hline & 3.2. Información sobre valoración, selección y eliminación & & & & \\
\hline & 3.3. Nuevos ingresos & & & & \\
\hline & 3.4. Sistemas de organización & & & & \\
\hline \multirow{5}{*}{ 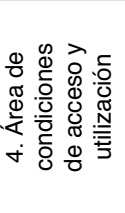 } & 4.1. Condiciones que rigen el acceso & & & & \\
\hline & 4.2. Condiciones que rigen la reproducción & & & & \\
\hline & 4.3. Lengua/escritura del material & & & & \\
\hline & 4.4. Características físicas y requisitos técnicos & & & & \\
\hline & 4.5. Instrumentos de descripción & & & & \\
\hline \multirow{4}{*}{ 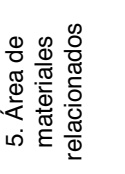 } & 5.1. Existencia y localización de originales & & & & \\
\hline & 5.2. Existencia y localización de copias & & & & \\
\hline & 5.3. Unidades de descripción relacionadas & & & & \\
\hline & 5.4. Nota de publicación & & & & \\
\hline 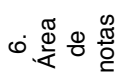 & 6.1. Notas & & & & \\
\hline \multirow{3}{*}{ 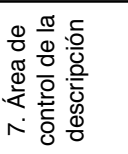 } & 7.1. Nota del archivero & & & & \\
\hline & 7.2. Reglas o convenciones & & & & \\
\hline & 7.3. Fecha de las descripciones & & & & \\
\hline
\end{tabular}

Tabla I. Estudio de los requisitos funcionales de la descripción archivística. (Elaboración propia) 
El estudio de la tabla nos permitirá ver en qué medida los elementos de la ISAD $(G)$ se ajustan a las funciones que se especifican en dicha norma. De este modo, podremos revisar en función de cada nivel de descripción, si todos los elementos son útiles y qué características tienen que cumplir para realizar una función específica, además de analizar si los elementos obligatorios de la descripción recogen todas las funciones que se especifican en la norma. Es decir, la tabla nos servirá de base para la elaboración de normas de contenido, ya que estas son las que tienen que tienen que reflejar el cumplimiento de cada función.

Teniendo en cuenta que no todos los elementos son obligatorios, podremos, a partir del estudio de los requisitos, de los objetivos, recursos y prioridades de cada centro, hacer una planificación de qué elementos entre los no obligatorios vamos a primar en la descripción.

\section{Notas}

(1) Normas de estructura: son las encargadas de establecer qué elementos son los que pueden formar parte de las distintas representaciones, sean descripciones de archivos, descripciones de productores o descripciones de funciones (Santamaría, 2003).

(2) Normas de contenido: destinadas a regular cómo debe formalizarse la información dentro de cada uno de los elementos anteriores (puntuación, uso de mayúsculas, formatos de fechas...) (ibidem).

(3) Normas de valores: encargadas de fijar cuáles son las formas de los nombres/términos o los códigos que están autorizados para su inclusión en un determinado elemento (ibidem).

(4) Normas de intercambio: destinadas a establecer los métodos que posibilitan que la información pueda ser compartida entre los sistemas informáticos de una o varias instituciones, es decir, los distintos protocolos. Y las de codificación de datos, que facilitan un formato común de intercambio o comunicación (ibidem).

(5) En nuestro caso nos vamos a centrar en la $\operatorname{ISAD}(G)$, por ser una norma más general y más ampliamente extendida.

(6) La norma recoge en la definición la función "explicar" con el sentido de contextualizar la información que se contiene en el documento. A partir de aquí sustituiremos "explicar" por "contextualizar" con el fin de aportar mayor claridad.

\section{Referencias}

Amat, C. B.; Pérez Salinas, I.; Ramos Pérez, A. (1989). Aplicación del modelo entidad relación a la definición de datos y al establecimiento de relaciones en el diseño de una base de datos de información de actualidad. Item. 5 (1989) 63-81.

Bearman, David (1993). Strategy for the Development and Implementation of Archival Description Standards. // Toward International Descriptive Standards for Archives: papers presented at the ICA Invitational Meeting of Experts on Descriptive Standards,

Scire. $11: 1$ (en.-jun. 2005) 103-112. ISSN 1135-3761. 
National Archives of Canada, Ottawa, 4-7 October 1988, Múnich; New Providence; Londres; París: K.G. Saur, 1993, 161-171 (ISBN 3-598-11163-0).

Bonal Zazo, José Luis. (2002) La descripción archivística normalizada: origen, fundamentos, principios y técnicas. Gijón: Trea, 2002.

Bonal Zazo, José Luis; Generelo Lanaspa, Juan José; Travesí de Diego, Carlos (2001). Manual de Descripción Multinivel: propuesta de adaptación de las normas internaciones de descripción archivística. Valladolid: Junta de Castilla y León, 2001.

Carnicer Arribas, Ma Dolores; Generelo Lanaspa, Juan José (1999). Hacia una aplicación uniforme de la ISAD (G): los niveles de la descripción. // Tábula. 4 (1999) 139-170.

CIA 88 Toward International Descriptive Standards: Papers presented at the ICA Invitational Meeting of Experts on Descriptive Standards, National Archives of Canada, Ottawa 4-7 October 1988 (1999). Compiled and edited with the financial assistance of the Toronto Area Archivists Group Education Foundation = Projet de normes internationales de description en archivistique: Communications présentées B la réunion restreinte d'experts en normes de description, Archives Nationales du Canada, Ottawa, du 4 au 7 octobre 1988 / Receuil rassablé et publié avec l'aide financière de la Toronto Area Archivists Group Education Foundation. - Múnich; New Providence; Londres; París: Saur, 1993. - ISBN 3-598-11163-0.

Fox, Michael J. (2001). Standarizing: locating EAD in the descriptive firmament. // Encoded Archival Description on the Internet. Nueva York: The Haworth Informacion Press, 2001.

Heredia Herrera, Antonia (1991). Archivística General: teoría y práctica. $5^{\text {a }}$ ed. Sevilla: Diputación Provincial de Sevilla, 1991.

ISAD (G): norma general internacional de descripción archivística preparada por la Comisión Ad Hoc de normas de descripción, Estocolmo, Suecia, 19-22 septiembre 1999. Madrid: Ministerio de Cultura, Dirección General del Libro, Archivos y Bibliotecas, 2000.

Ríos Hilario, Ana Belén (2003). Nuevos horizontes en el análisis de los registros y la normativa bibliográfica. Gijón: Trea, 2003.

Santamaría Gallo, Abelardo (2003). Nuestras normas nacionales de descripción archivística en el marco internacional. // Archivamos. 47-48 (2003) 5-14.

Walch, Victoria Irons (1994). Standards for Archival Description: a handbook. Chicago: Society of American Archivists, 1994. 